\section{Videojuego Educativo como Herramienta de Apoyo para la Enseñanza del Idioma Inglés "Philip's Adventure"}

\section{Educational Video Game as a Support Tool for the Teaching of the English Language "Philip's Adventure".}

\section{Juan Carlos Herrera Estrada}

Maestría Gestión de Tecnologías de la Información

(En Curso). Universidad Minuto de Dios

Docente TCO. Cundinamarca. Colombia.

juan.herrera.e@uniminuto.edu

ORCID https://orcid.org/oooo-0oo2-5640-9257

\section{Resumen}

El uso de videojuegos como herramientas en el aula de clase, es una actividad que poco se aplica en las instituciones educativas, pero que promete un significativo resultado para los estudiantes, puesto que permite a éstos cambiar de rutina, así como explorar contenidos temáticos de forma divertida. Si se utilizan videojuegos para la enseñanza de temas que poco gustan a los estudiantes, como las matemáticas y el inglés, éstos se convierten en una poderosa herramienta, que además de ser útil para el docente, tornan el ambiente más dinámico y agradable, motivando a los educandos a aprender.

Teniendo en cuenta lo anterior, se decidió desarrollar éste proyecto en el cual se implementa un videojuego que sirva como herramienta de apoyo para la enseñanza del idioma inglés, dirigido a los estudiantes de cuarto

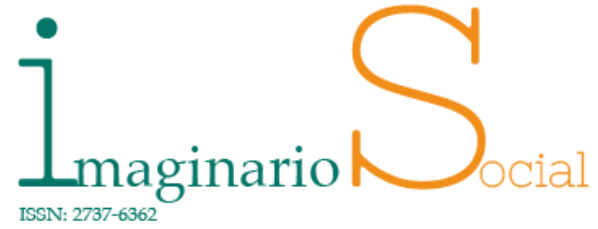

Imaginario Social e-ISSN: 2737-6362

julio - diciembre 2020 Vol. 3-2-2020 http://revista-

imaginariosocial.com/index.php/es/in dex

Recepción: 23 de abril 2019

Aceptación: 30 de abril 2020

18-39

Atribución/Reconocimiento-NoCo mercial- Compartirlgual 4.0 Licencia Pública Internacional - CC BY-NC-SA 4.0 https://creativecommons.org/licenses /by-nc- sa/4.0/legalcode.es 
primaria del Colegio Gimnasio de los Andes ubicado en el municipio de Ubaté.

Palabras clave: Videojuegos, inglés, idioma, aprendizaje, educación, herramientas de apoyo, institución educativa, estrategias, estudiantes.

\begin{abstract}
The use of video games as tools in the classroom, is an activity that is little applied in educational institutions, but that promises a significant result for students, since it allows to change routine, as well as explore content theme in a fun way. If video games are used to teaching subjects that very little like students, such as mathematics and English, they become a powerful tool, that in addition to being useful for the teacher, make the environment more dynamic and pleasant, encouraging learners to learn. Taking into account the above, it was decided to develop this project, which implements a video games that serves as a support tool for the teaching of the language English, intended for students of the fourth primary school gym of de Andes located in the municipality of Ubate
\end{abstract}

Key word: Video games, English, language, learning, education, support, educational institution, strategies, student tools.

\title{
Introducción
}

El inglés, un idioma hablado en más de 100 países, y catalogado como el lenguaje universal, se ha convertido en una herramienta de gran valor para los países de habla hispana como Colombia, en donde para sus habitantes, hablar este idioma representa tener más oportunidades y además un valor agregado que les permita competir con otros profesionales.

Por lo anterior las instituciones educativas como escuelas, colegios y universidades se han esforzado, en que el inglés tenga una gran aceptación por parte de los estudiantes. Sin embargo, las estrategias de enseñanza utilizadas para cumplir con este objetivo no siempre resultan efectivas para muchos de ellos, esto se evidencia en estudios realizados por el CEER y la organización Education First, los cuales se detallarán más adelante. 
Siendo evidente el bajo nivel de inglés que presentan las instituciones educativas se decidió elaborar un videojuego que sirva como herramienta de apoyo para el docente en la enseñanza del idioma inglés, ya que el uso de juegos resulta ser un método que además de divertir logra motivar y estimular a los estudiantes para que aprendan.

Estudios realizados recientemente por el CEER en Colombia y por Education First, a nivel mundial, demuestran el bajo nivel que enfrenta el país en cuanto al conocimiento y habla del idioma inglés. El estudio realizado y publicado en el 2013 por el CEER, se basó en el análisis de las pruebas Saber 11 y Saber Pro, dejando entrever el bajo nivel de inglés que tienen los estudiantes en el país. El otro estudio, realizado por la organización Education First en el año 2014, a través de un ranking de dominio del inglés, ubica a Colombia en el lugar 46 de entre 60 países.

Este problema existe desde hace mucho tiempo y se genera a partir de los primeros años de estudio en las escuelas de educación básica primaria, en donde todavía no existen estrategias adecuadas de enseñanza, que logren incentivar a los estudiantes a acercarse al idioma inglés.

Otro factor a tener en cuenta son las creencias que existen en nuestra sociedad, acerca del alto grado de dificultad que representa el aprender dicho idioma, lo cual genera desinterés por parte de los estudiantes.

Con el fin de evaluar la situación de manera más concreta, en el mes de junio del año 2014, se evaluó a un grupo de estudiantes de grado cuarto de primaria del colegio Gimnasio de los Andes, de la Provincia de Ubaté. A este grupo, conformado por 18 estudiantes, se le realizó una prueba de nivel de inglés escrita o "placement test", diseñada por la Universidad de Cambridge, la cual tiene como base el Marco Común Europeo de Referencia para Lenguas y cubre los dos primeros niveles de inglés (Principiante y Elemental). Los resultados generales del test indican que todos los estudiantes examinados se ubican en el nivel Principiante.

De igual manera, se realizaron encuestas de opinión, dirigidas al docente del área de inglés y a los 18 estudiantes del grado cuarto de primaria, con el fin de visualizar la viabilidad del videojuego, el grado de aceptación de los estudiantes y la adaptación de las metodologías de enseñanza del docente de inglés al proyecto. Como era de esperarse, el análisis de los resultados de la encuesta realizada a los estudiantes, indica que la mayoría de estudiantes están de acuerdo en que las clases serían más divertidas 
con el uso de un videojuego, así mismo se evidencio su gran interés por participar en el proceso de desarrollo del proyecto.

¿De qué manera un videojuego puede apoyar el proceso de enseñanza del idioma inglés en el grado cuarto de primaria del colegio Gimnasio de los Andes?. Como objetivo se pretende implementar un videojuego educativo como herramienta de apoyo para la enseñanza del idioma inglés en el Colegio Gimnasio de los Andes.

En los últimos años se han realizado grandes avances en el área de las telecomunicaciones (Internet, redes sociales, smartphones, entre otros), lo cual ha permitido el acercamiento de las personas en todo el mundo, haciendo cada vez más evidente la necesidad de utilizar un lenguaje universal. El inglés es el idioma que más se aproxima a esta definición, gracias a su amplio uso a nivel mundial. Además de esto, el Consejo Británico indica que un tercio de los libros en el mundo son publicados en inglés y que el 75 \% de la bibliografía científica también está en inglés. Por esto resulta indispensable para cualquier profesional el aprender inglés, pues de ésta manera tendrá acceso a libros especializados y a los últimos avances en su área del conocimiento (Crystal, 2003).

Teniendo en cuenta lo anterior, se pasará a indagar un poco sobre la situación actual en Colombia. Recientemente, el CEER publicó un estudio el cual revela que tan sólo el 2,4 \% de los bachilleres del país tiene buenos niveles de inglés, al igual que el $6 \%$ de los universitarios. Estos porcentajes son producto de análisis realizados a los resultados de las pruebas nacionales Saber 11 y Saber Pro, en donde se tuvo en cuenta el Marco Común Europeo de Referencia para Lenguas. Esto refleja el bajo nivel de inglés existente en el país, empezando por la escuela básica primaria, en donde se debe asentar las bases para una comunicación efectiva en inglés.

En un plano más local, los autores de éste proyecto estudiaron la situación de uno de los colegios de la Provincia de Ubaté en donde se seleccionó al colegio Gimnasio de los Andes por su cercanía y además por la disponibilidad de sus directivos y docentes para trabajar en proyectos de mejoramiento de la enseñanza. Se trabajó con el grupo de estudiantes de grado cuarto de básica primaria a solicitud de la docente de inglés Mercedes Robayo, dicho grupo está conformado por niños con una edad promedio de diez años. 
En el presente proyecto se propone la implementación de un videojuego educativo como respuesta a la necesidad de encontrar una nueva estrategia de apoyo a la enseñanza del inglés desde el salón de clase, la cual resulte atractiva y capte la atención de los estudiantes. Con el uso del videojuego se pretende incentivar a los estudiantes de educación básica primaria del colegio para que aprendan el idioma, además de conseguir implantar en ellos los conocimientos necesarios para dominar esta segunda lengua de una manera práctica, natural y a la vez entretenida.

Asimismo, tanto el profesor del área de inglés como los alumnos de grado cuarto de primaria, estarían involucrados de forma activa en los procesos de mejora y evaluación del videojuego.

\section{Materiales y Métodos}

Las actividades y productos esperados están basados en la metodología SUM (Ver Metodología de desarrollo).

Tabla 1. Diseño metodológico.

Objetivo general: Implementar un videojuego educativo como herramienta de apoyo para la enseñanza del idioma inglés en el Colegio Gimnasio de los Andes.

\begin{tabular}{lllll}
\hline $\begin{array}{l}\text { Objetivos } \\
\text { específico }\end{array}$ & Actividades & Técnicas & $\begin{array}{l}\text { Instrumento } \\
\text { S }\end{array}$ & $\begin{array}{l}\text { Producto } \\
\text { esperado }\end{array}$ \\
\hline $\begin{array}{l}\text { Recopilar } \\
\text { los }\end{array}$ & $\begin{array}{l}\text { Reuniones } \\
\text { periódicas con }\end{array}$ & $\begin{array}{l}\text { Entrevista no } \\
\text { estructurada. }\end{array}$ & Cuestionario. & Pila \\
requisitos & la docente del & & del \\
del & área de inglés & & Documento del \\
videojuego & para definir los & & \\
con el fin de & elementos que & & \\
crear un & componen el & & \\
concepto & concepto del & & \\
claro del juego. & & & \\
mismo, y & & & \\
definir & & & \\
aspectos & & & \\
clave para la & & & \\
fase & & & \\
diseño. & & & & \\
\end{tabular}




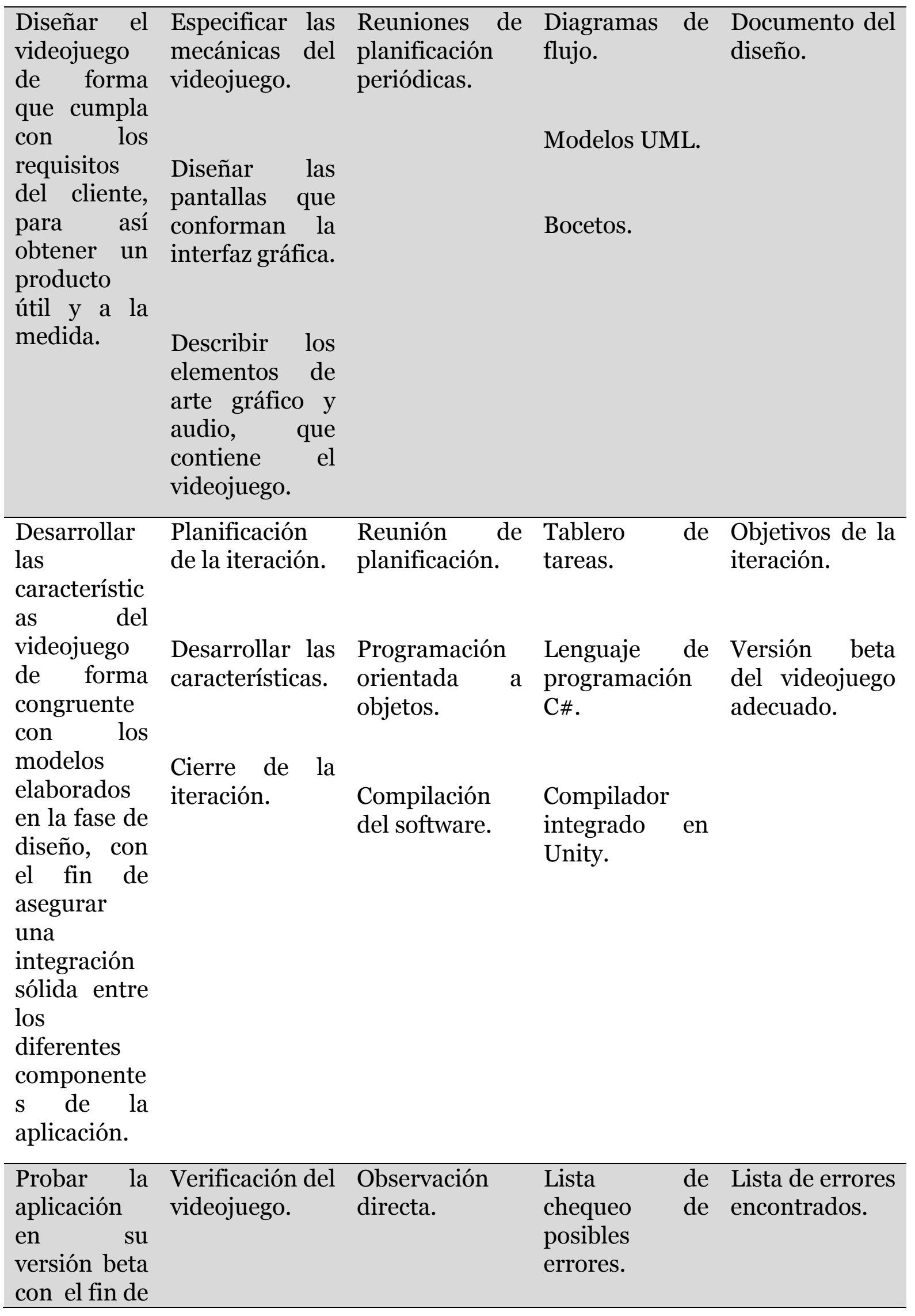




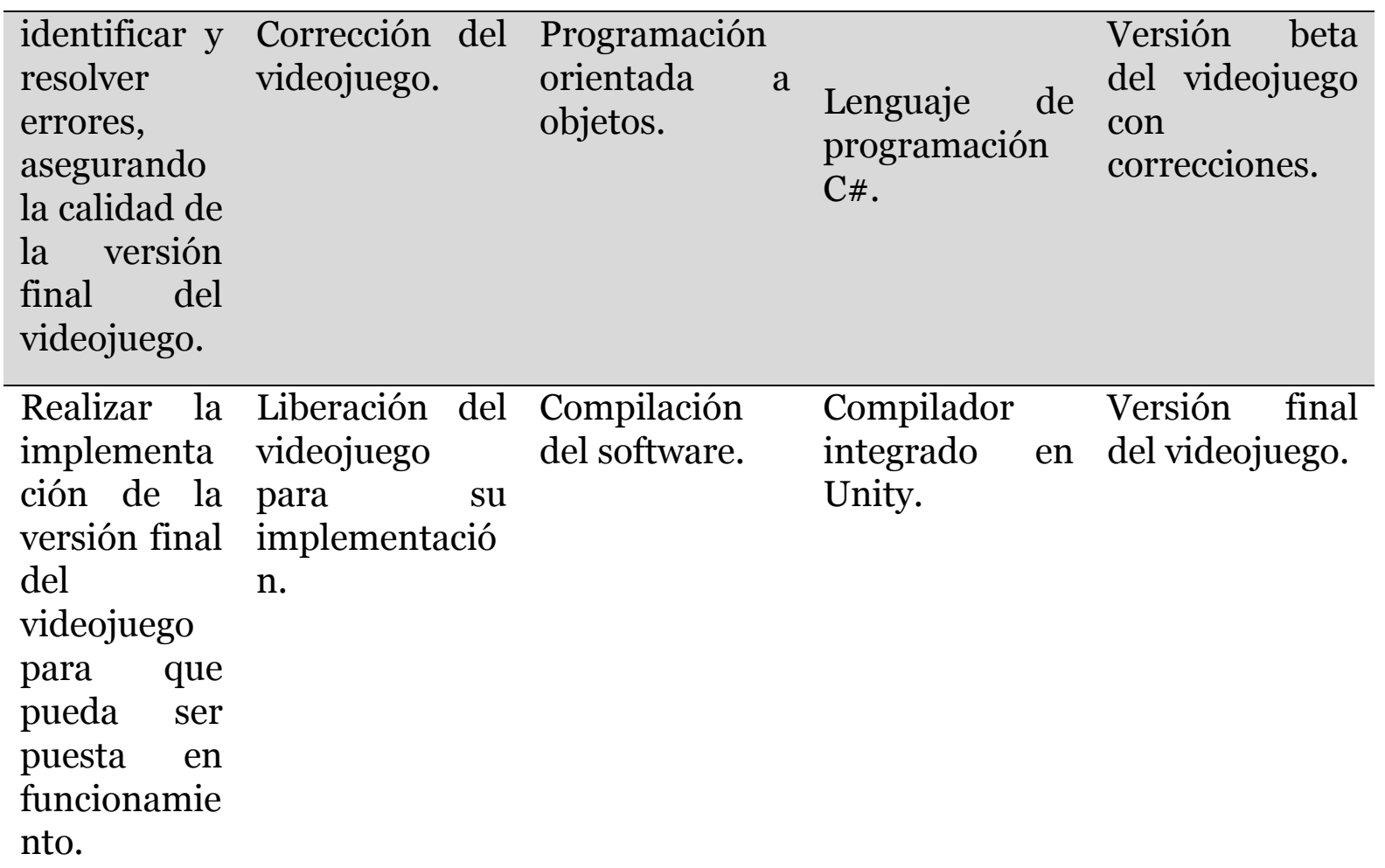

\section{Metodología SUM}

Para el desarrollo del presente trabajo se utilizará SUM, una metodología para la creación de videojuegos, que tiene como base las metodologías ágiles de desarrollo de software Scrum y XP.

La metodología SUM para videojuegos tiene como objetivo desarrollar videojuegos de calidad en tiempo y costo, así como la mejora continua del proceso para incrementar su eficacia y eficiencia. Pretende obtener resultados predecibles, administrar eficientemente los recursos y riesgos del proyecto, y lograr una alta productividad del equipo de desarrollo. SUM fue concebida para que se adapte a equipos multidisciplinarios pequeños (de tres a siete integrantes que trabajan en un mismo lugar físico o estén distribuidos), y para proyectos cortos (menores a un año de duración) con alto grado de participación del cliente (Acerenza N., et al., 2009).

\section{Roles de SUM}

Se definen cuatro roles: equipo de desarrollo, productor interno, cliente y verificador beta. El productor interno y el cliente se corresponden en forma directa con los roles de Scrum Master y Product Owner de Scrum respectivamente. 
El equipo de desarrollo tiene las características del Scrum team, pero a diferencia de Scrum se definen subroles dentro del equipo. Es necesario esta definición ya que se requiere una alta especialización para satisfacer las distintas disciplinas que involucra el desarrollo de videojuegos, aspecto no contemplado en Scrum.

El rol de verificador beta no está presente en Scrum pero sí se detecta su existencia en el relevamiento de la realidad local y en la industria del videojuego en general. $\mathrm{Su}$ responsabilidad es la de realizar la verificación funcional del videojuego y comunicar su resultado. Sin embargo puede no poseer experiencia ni ser jugador frecuente y participar igualmente de la verificación, por ejemplo, al formar parte de un focus group del videojuego. (Acerenza N., et al., 2009)

Los roles en el presente proyecto se identifican de la siguiente manera:

- Product owner: Profesor del área de inglés del colegio Gimnasio de los Andes.

- Scrum Master: Ingeniero Juan Carlos Herrera (Director del Proyecto).

- Scrum Team: Felipe Andres Lopez, Diego Alexander Mellizo.

- Verificador Beta: Estudiantes de cuarto grado del Colegio Gimnasio de los Andes.

\section{Ciclo de Vida de SUM}

En (Acerenza N., et al., 2009), el ciclo de vida se divide en fases iterativas e incrementales que se ejecutan en forma secuencial con excepción de la fase de gestión de riesgos que se realiza durante todo el proyecto. Las cinco fases secuenciales son: concepto, planificación, elaboración, beta y cierre.

Las fases de concepto, planificación y cierre se realizan en una única iteración, mientras que elaboración y beta constan de múltiples iteraciones. Las fases surgen como adaptación al desarrollo de videojuegos de las fases pre-game, game y post-game que presenta Scrum, donde las dos primeras coinciden con las fases de planificación y elaboración, mientras que la tercera se corresponde con las fases de beta y cierre.

Esta división se realiza ya que la fase beta tiene características especiales en la industria de videojuegos. La fase de concepto no se corresponde con ninguna etapa de Scrum y se agrega ya que cubre necesidades específicas para el desarrollo de videojuegos y se identifica su uso en la realidad local y en la industria mundial. 


\section{Resultados}

\section{Bocetos.}

A continuación se presentan los bocetos realizados para el escenario y cada una de las misiones que integrarán el videojuego.

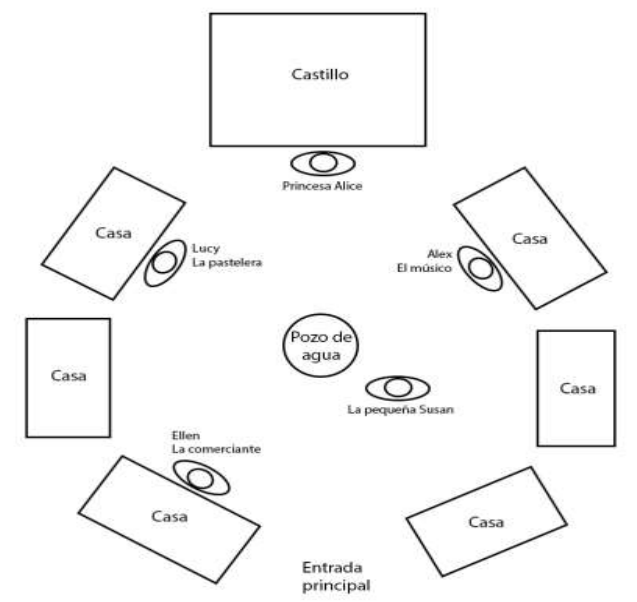

Figura 1. Boceto de Ciudad Medieval.

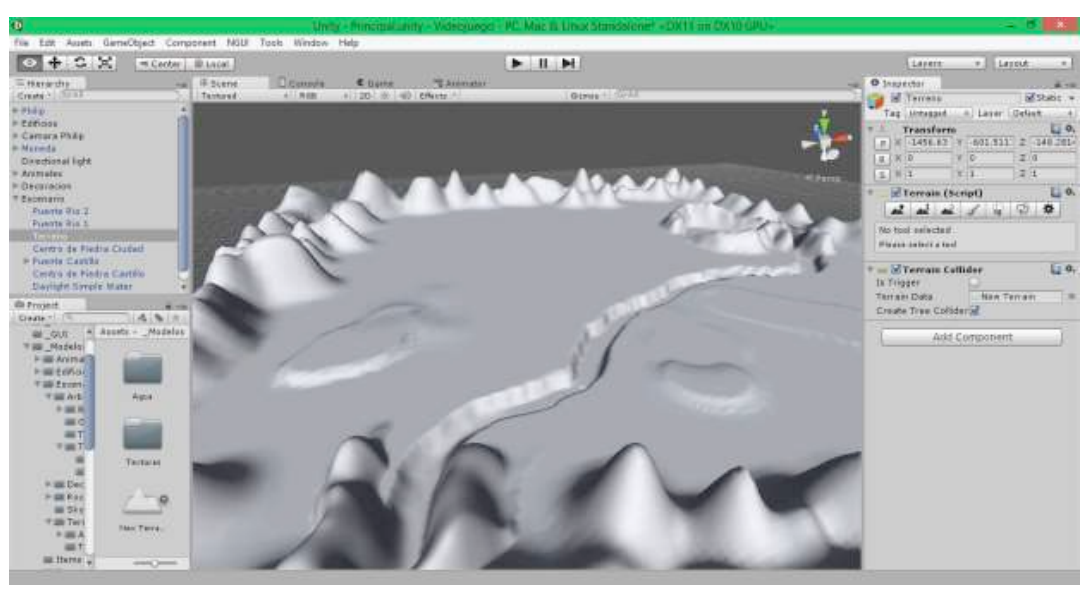

Figura 2. Modelado del terreno para el videojuego. 

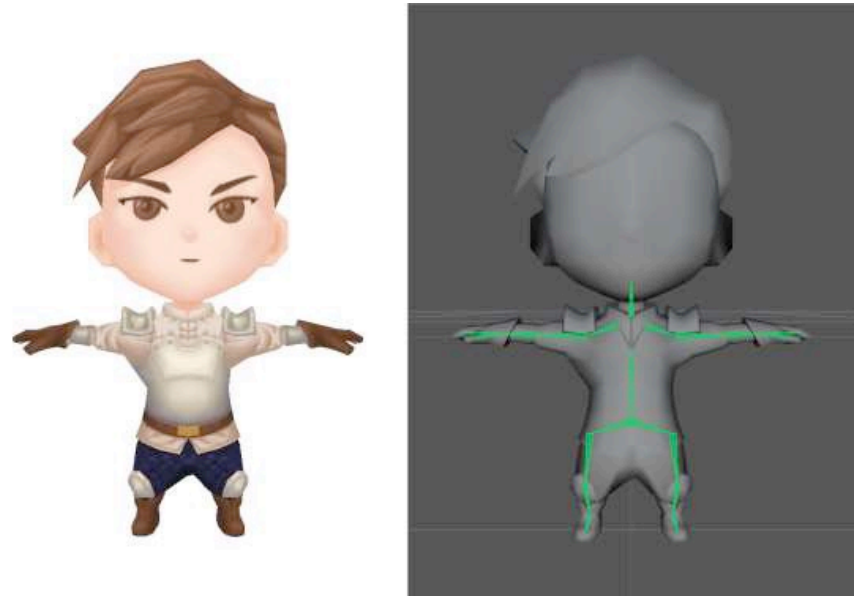

Figura 3. Personaje principal. Modelo en 3D (izquierda) y esqueleto (derecha).

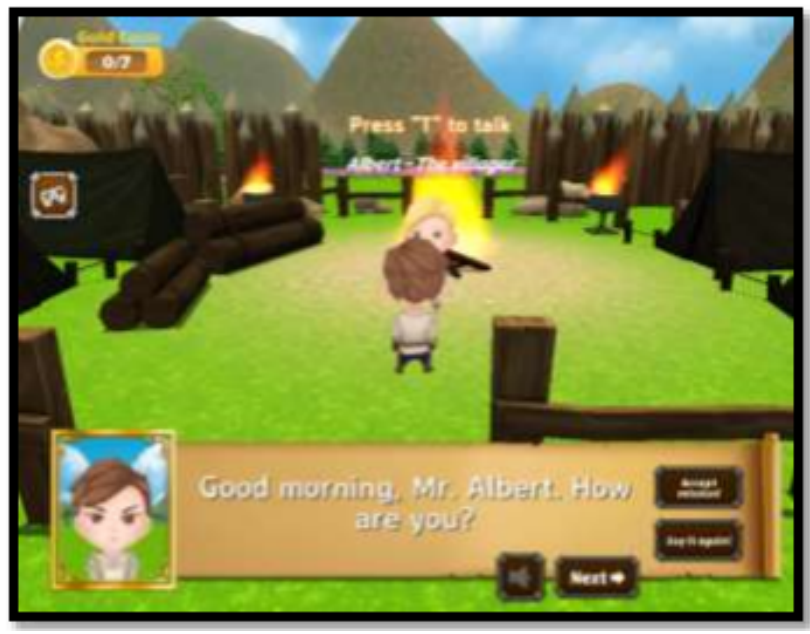

Figura 4. Misión 1(estado inicial)

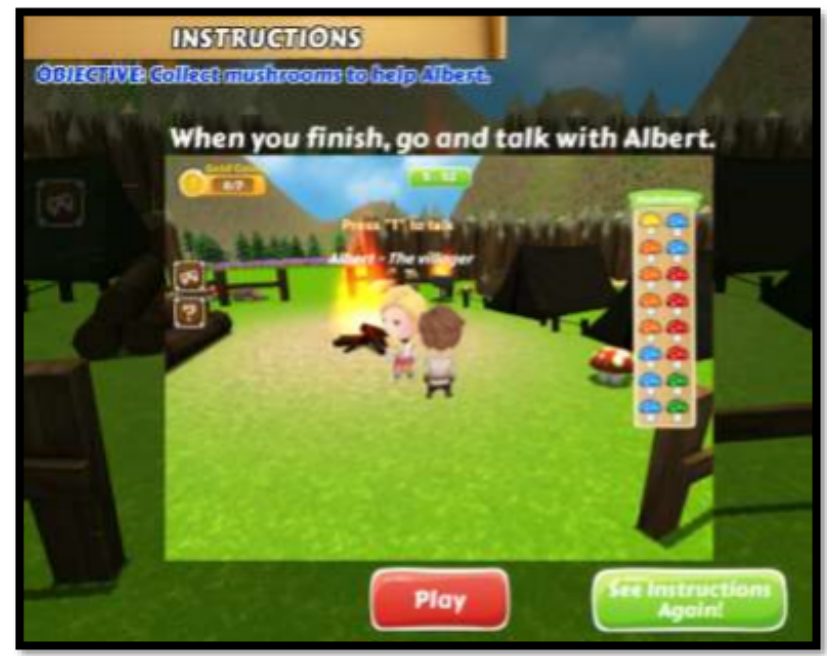

Figura 5. Instrucciones misión 1 


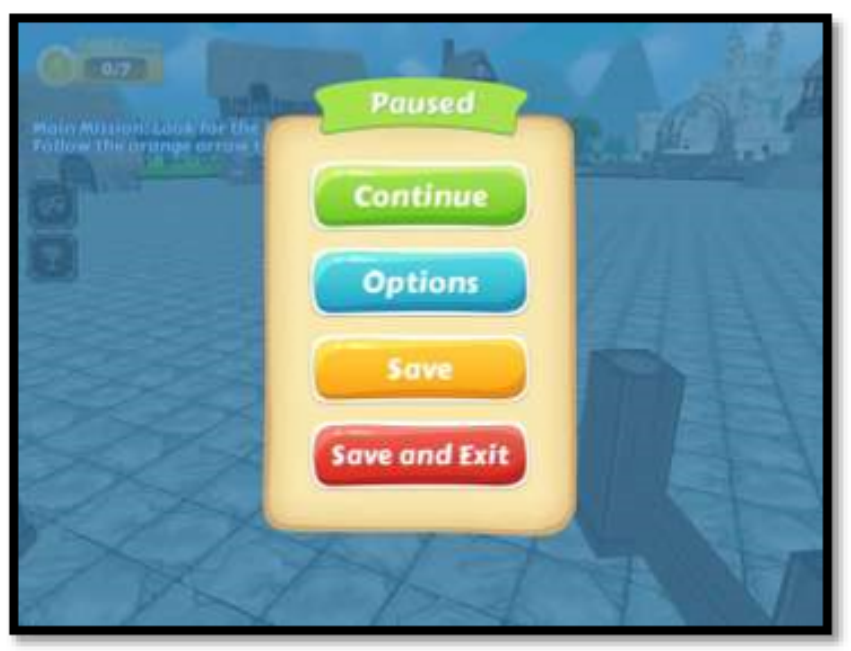

Figura 6. Menú de Pausa

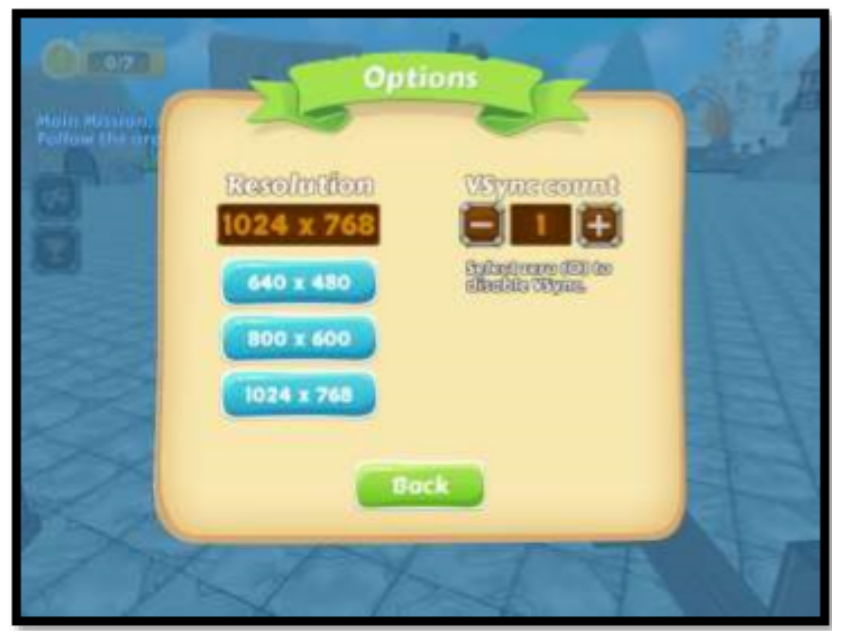

Figura 7. Opciones Graficas del Juego

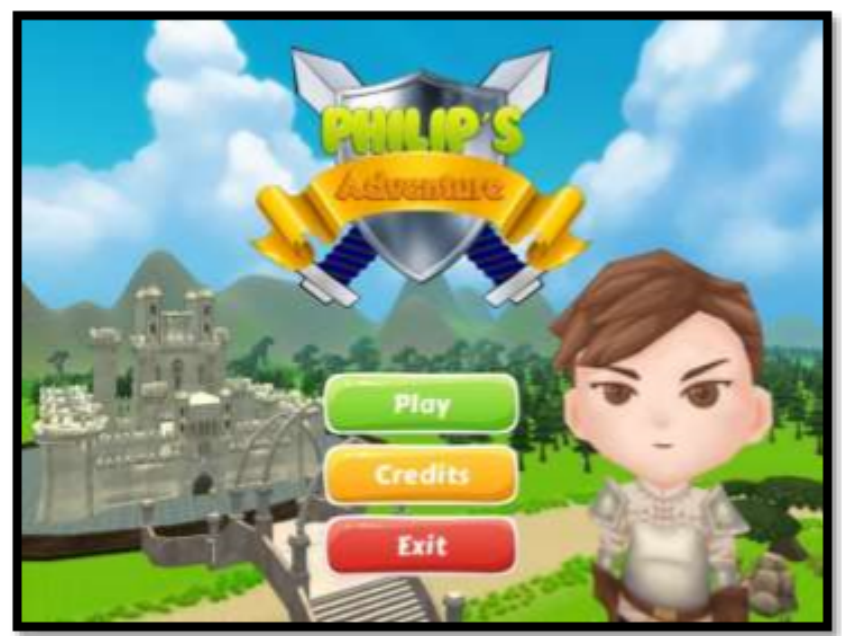


Figura 8. Pantalla Main Menu

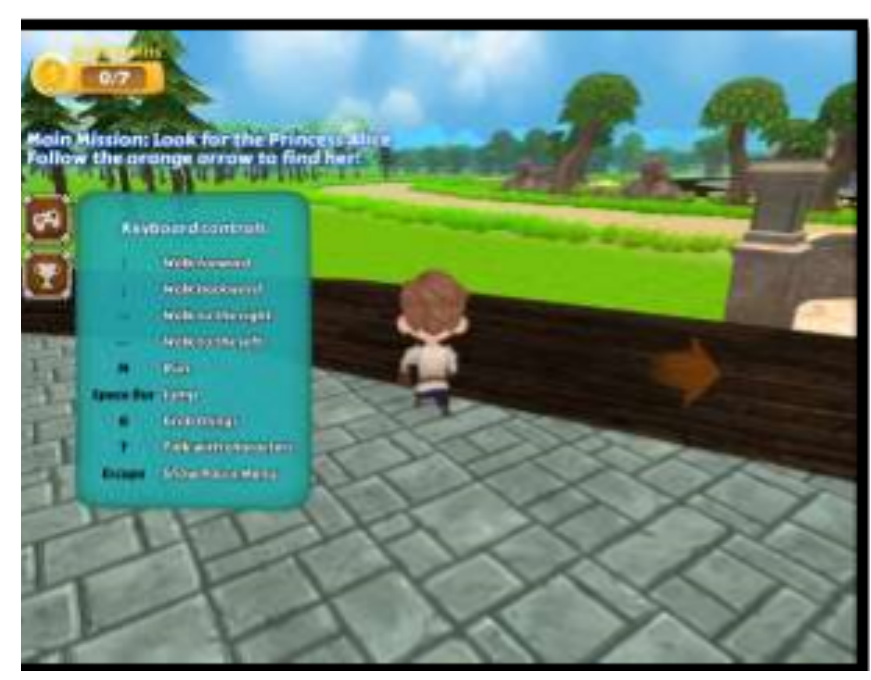

Figura 9. Panel de Control

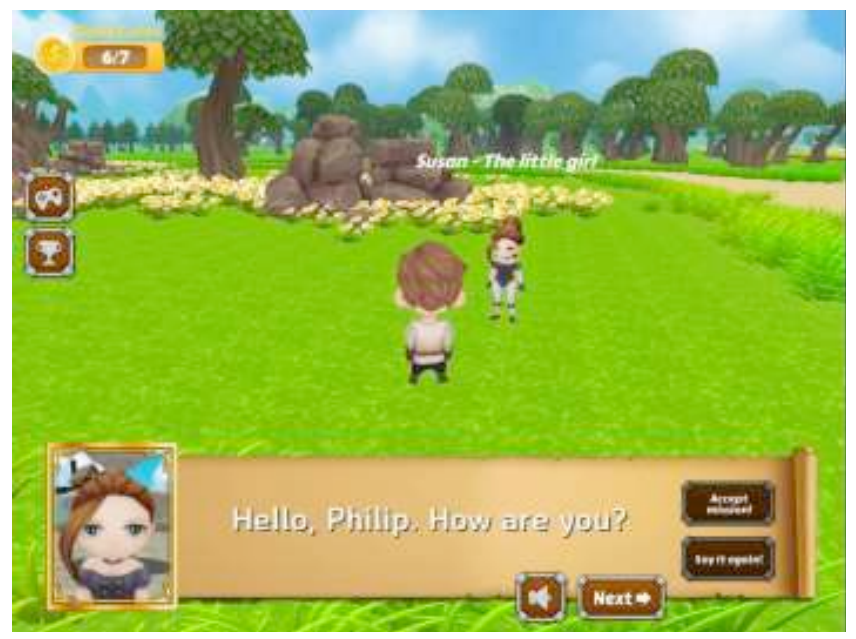

Figura 10. Misión 7 (estado inicial).

En la Figura 11 se encuentra Ciudad Medieval, lugar donde Philip llevará a cabo la mayoría de sus misiones. Al fondo se puede observar el castillo de la Princesa Alice. 


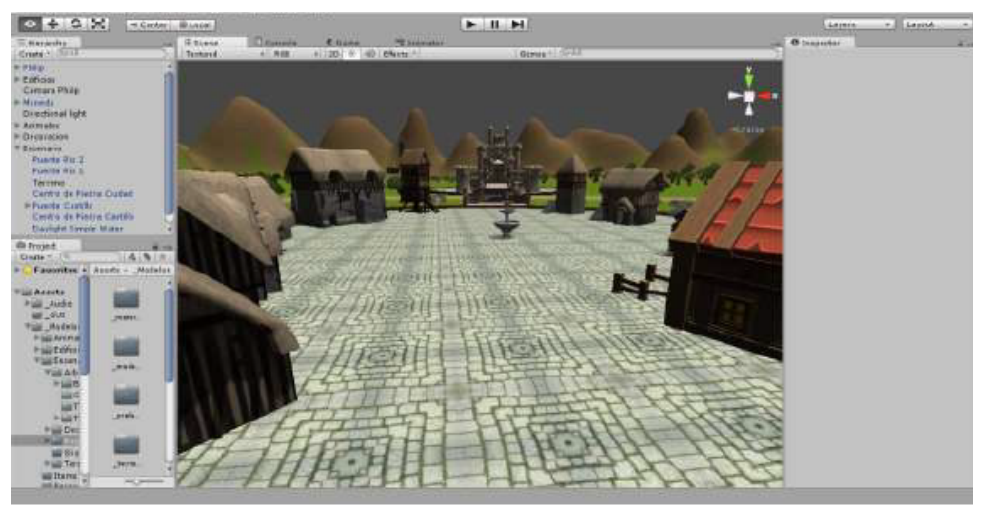

figura 11. ciudad medieval.

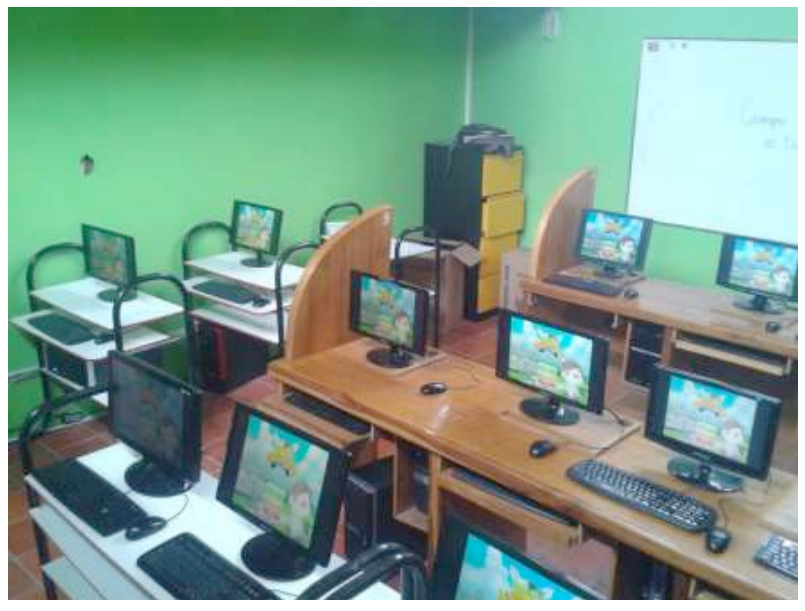

Imagen 1. Implementación de la versión beta 1.o del videojuego

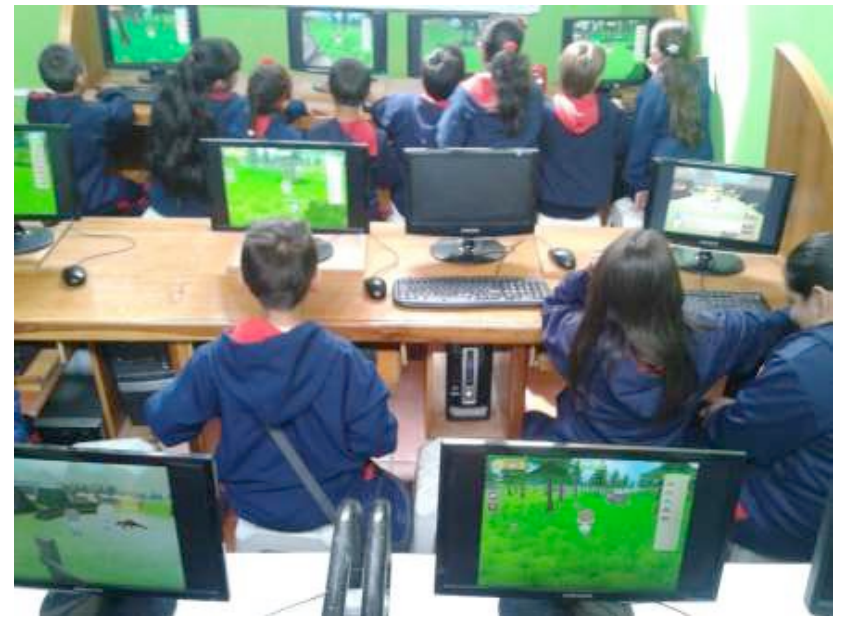

Imagen 2. Pruebas con estudiantes de cuarto de primaria 


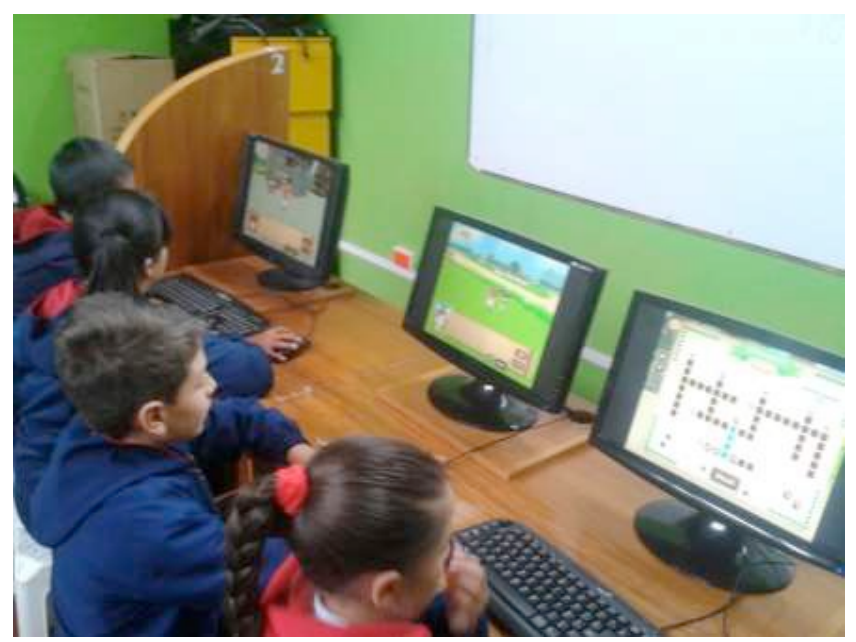

Imagen 3. Pruebas con estudiantes de cuarto de primaria

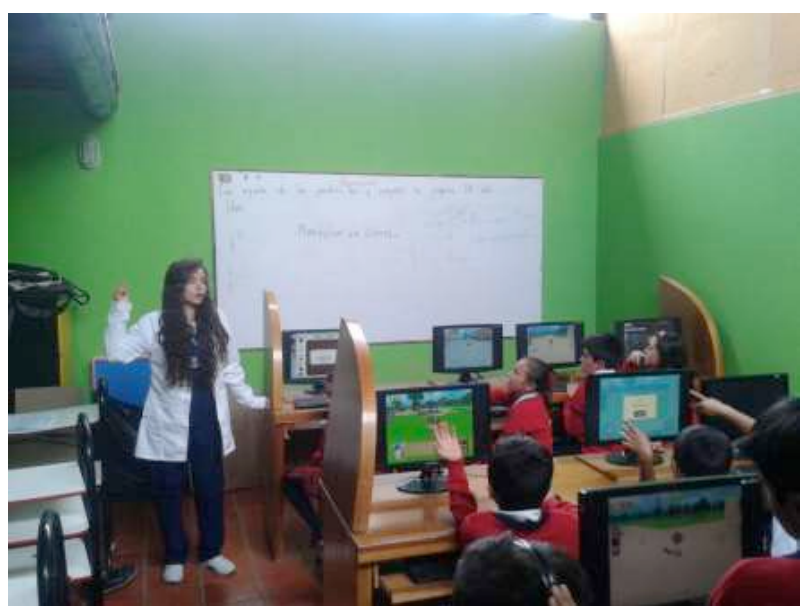

Imagen 4. Pruebas con estudiantes de cuarto de primaria

\section{Lista de errores encontrados.}

Durante las diferentes pruebas realizadas a la versión 1.0 del videojuego, se encontraron los siguientes errores:

- El videojuego se ejecuta, pero al cargar la escena se muestra un error y se interrumpe la ejecución del mismo (sólo en algunos computadores).

- El personaje principal es muy lento al caminar.

- El personaje principal gira muy rápido.

- Hay poco tiempo en algunas misiones.

\section{Corrección de errores.}

El error con mayor prioridad para ser resuelto fue el relacionado con los computadores en los que la ejecución del videojuego se interrumpía; en este caso se realizó una 
revisión de las especificaciones técnicas de cada uno de los computadores, en donde se encontró que los mismos tenían una característica en común, utilizaban la misma referencia de tarjeta gráfica (modelo Intel GMA 4500), a través de búsquedas en la Internet y de revisar las especificaciones técnicas de dicha tarjeta se determinó que este modelo de tarjeta no soporta una característica denominada vertex shader, a continuación se sustituyeron los shaders del juego que hacían uso de dicha característica, lo cual resolvió el problema.

Cada uno de los errores de la lista fue solucionado y se obtuvo la versión final 1.1 del videojuego (versión beta 1.o con errores corregidos), la cual se compiló con éxito.

\section{Discusión}

En Colombia son escasos los proyectos en donde se hayan desarrollado videojuegos como herramientas para el aprendizaje, especialmente aquellos referentes al inglés. Dentro de los proyectos de este tipo más relevantes destaca uno realizado en conjunto entre la Universidad Nacional [UN] y la Universidad de Caldas, el cual se detalla a continuación:

Por medio de un juego de video adaptado al contexto local, los niños podrían aprender inglés de manera lúdica y al mismo tiempo aumentar sus destrezas en el área de sistemas. Angelo Adrian Quintero Ciprián, estudiante de Administración de Empresas de la UN en Manizales y un grupo de jóvenes emprendedores de la Universidad de Caldas, desarrollaron el proyecto Tiny Town para que los niños aprendan el idioma inglés jugando. Este proyecto ya cuenta con un prototipo probado en un grupo de niños, quienes en el juego mostraron la apropiación de los conceptos enseñados e hicieron sugerencias sobre el entorno de juego, las misiones que deben superar y los personajes.

A partir de los estándares pedagógicos del Ministerio de Educación desarrollaron un software a manera de video juego, en el cual los niños pueden interactuar entre ellos y con personajes animados deben superar diferentes obstáculos para llegar a la meta, y en cada nivel van aprendiendo elementos básicos del idioma que se van complejizando a medida que avanza el juego. 
"El Ministerio propone unos estándares básicos dependiendo del grado escolar en el que se va aplicar. Hay unas directrices de los conceptos que se deben enseñar para determinada edad, nosotros nos basamos en ello para crear la dinámica del juego y los escenarios virtuales en los que el niño debe desenvolverse para que se efectúe el aprendizaje", explicó Quintero Ciprián.

\section{Objetivo educativo general del videojuego.}

Philip's Adventure es un videojuego que se adapta a la metodología de enseñanza CLT usada por la docente del área de inglés para dictar sus clases, la cual está enfocada en el aprendizaje de una segunda lengua.

En el videojuego, se refuerzan las habilidades de lectura, escritura y escucha. Se muestran los contenidos de forma interactiva como juegos de cartas, juegos de palabras, rondas infantiles y crucigramas, atendiendo a las temáticas que se trabajan en el grado cuarto.

A continuación se describirán los componentes del videojuego en los cuales se implementaron las temáticas del grado cuarto y la metodología de enseñanza CLT:

Sistema de diálogos: en los diálogos se usa la metodología CLT o Enseñanza Comunicativa de la Lengua (para más información ver Marco Conceptual), la cual insiste en la comunicación como el pilar para lograr la comprensión de una segunda lengua, este aspecto se manifiesta cuando Philip (personaje principal) debe establecer una comunicación con otros personajes y asumir un papel en cada situación que se le presenta. Siempre habrá una necesidad de comunicación en el videojuego, si Philip no se comunica con algún personaje para recibir instrucciones, no podrá cumplir su objetivo.

El sistema de diálogos también incluye temáticas como: Wh questions, Yes/No questions, Can, simple present, continuous present, short answers, affirmative $y$ negative.

Misiones: En las misiones se implementan ayudas didácticas como crucigramas, juegos de cartas, canciones infantiles en inglés y textos, que la docente de inglés usa como herramientas de apoyo dentro de sus clases y son de gran ayuda para mantener a los estudiantes activos y motivados. 


\section{Documento de concepto.}

\section{Visión.}

Philip's Adventure es un videojuego en 3D de un sólo jugador, en el cual conocerás a la gente que vive en Ciudad Medieval y cumplirás desafíos con el fin de convertirte en un Caballero Medieval. Tú tomarás el papel de Philip, un joven aventurero con deseos de aprender muchas cosas nuevas.

\section{Género.}

Es un videojuego de rol en tercera persona. El jugador controlará a Philip, el personaje principal, el cual deberá cumplir una serie de misiones con el fin de lograr su objetivo de convertirse en Caballero Blanco.

\section{Mecánica de juego.}

La mecánica del juego consiste en controlar al personaje principal, Philip, quien puede moverse por todo el escenario en busca de las siete monedas de oro, que inicialmente pertenecerán a los habitantes de Ciudad Medieval. Cada habitante posee una moneda de oro, Philip deberá hablar con cada uno de ellos y a continuación recibirá instrucciones de lo que deberá hacer para obtener la moneda. El juego no tiene niveles de dificultad y este se lleva a cabo sobre un mismo y único escenario.

\section{Características.}

- Diversidad de Misiones: las misiones tienen contenidos de diversa temática y jugabilidad.

- El entorno está rodeado de naturaleza como árboles, agua, montañas y animales.

- Interacción del personaje principal con los personajes secundarios.

- El idioma del videojuego esta sólo en inglés.

\section{Ambientación.}

El lugar donde Philip lleva a cabo su aventura es un único escenario con objetos al estilo de la edad media como las casas, los personajes y la interfaz gráfica de usuario.

\section{Historia.}

Philip es un joven habitante de Ciudad Medieval, que sueña con llegar a ser un Caballero Blanco. Un día decide hablar con la Princesa Alice, líder de los Caballeros Blancos, quien le dice que para lograrlo tiene que llevarle 7 monedas de oro, las cuáles debe conseguir ayudando a los pobladores de Ciudad Medieval en sus actividades 
diarias y superando los desafíos que ellos le planteen, cada ciudadano le entregará a Philip una moneda de oro como recompensa.

\section{Misiones.}

Misión principal.

Philip tiene la misión de buscar a la Princesa Alice. Una vez que la encuentra, ella le dice que para convertirse en Caballero Blanco tienen que conseguir 7 monedas de oro, la cuáles debe obtener ayudando a los pobladores de Ciudad Medieval. Una vez Philip reúne las monedas, debe hablar de nuevo con la Princesa Alice, quién lo espera para nombrarlo Caballero Blanco.

Para completar esta misión es necesario realizar primero todas las misiones secundarias.

\section{Misiones secundarias.}

Las misiones secundarias inicialmente deben ser realizadas en orden secuencial. Al completar cada una de estas, Philip recibirá una moneda de oro, se desbloqueará la siguiente misión y podrá jugar nuevamente la misión superada.

\section{Conclusiones}

La entrevista utilizada para la recopilación de requerimientos, resultó ser un método efectivo, puesto que sirvió como una guía para especificar de forma organizada los requerimientos del cliente, permitiendo así una clara definición de las características del videojuego a diseñar.

A través de instrumentos tales como bocetos, modelos y diagramas, se logró diseñar el videojuego acorde a los requisitos exigidos por el cliente, asegurando el desarrollo de un producto a la medida.

Gracias al desarrollo iterativo de las características, y mediante el uso de las plataformas de desarrollo seleccionadas, se logró una sólida integración de los módulos del videojuego y se redujo la posibilidad de que surgieran defectos.

La utilización de listas de chequeo para probar el videojuego, resultó ser un instrumento apropiado, el cual permitió la revisión del videojuego en su totalidad y la identificación de errores que posteriormente fueron corregidos, asegurando así la calidad del producto final. 
La implementación del videojuego se realizó exitosamente, asegurando su correcto funcionamiento, así como su utilización por parte de la docente de inglés como herramienta para orientar sus clases.

El videojuego implementado es considerado por la docente de inglés como una herramienta de apoyo efectiva, puesta que le permite evaluar el nivel de conocimientos de los estudiantes, así como identificar falencias respecto a los contenidos temáticos de inglés del grado cuarto.

A través de la revisión de la teoría relativa a los videojuegos, se puede concluir que existen numerosas maneras para adaptar las metodologías de enseñanza y los contenidos temáticos de inglés en forma de videojuego, esto debido a la gran cantidad de tipos de juego y herramientas de desarrollo que existen actualmente.

Los videojuegos de tipo rol resultan ser muy flexibles, permitiendo su unión con cualquier otro tipo de videojuego, además promueven la interacción social en el idioma inglés a través de la simulación de un entorno real.

Se logró entender la complejidad que requiere el desarrollar un videojuego, siendo una actividad multidisciplinaria, la cual requiere de artistas, diseñadores, sonidistas, guionistas y programadores, en donde cada miembro del equipo es de vital importancia para el éxito del proyecto.

\section{Referencias}

Aarseth, E. (2007). "Investigación sobre juegos: aproximaciones metodológicas al análisis de juegos”. En: Artnodes, Vol 7, Barcelona, 4-14.

Abrahamsson, P., et al. (2002). Agile Software Development Methods. VTT Publications.

Aceranza, N., et al. (2009). Una metodología para desarrollo de videojuegos: versión extendida. Montevideo, Uruguay: Universidad de la Republica.

Agencia de Noticias Universidad Nacional [UN]. (2011). Desarrollan video juego para aprender inglés. Recuperado de http://www.agenciadenoticias.unal.edu.co/ndetalle/article/desarrollan-videojuego-para-aprender-ingles.html 
Alegsa. (s. f.). Definición de Pipeline. Recuperado de http://www.alegsa.com.ar/Dic/pipeline.php

Arrioja, N. (2013). Unity: Diseño y Programación de Videojuegos. Buenos Aires. Argentina: Users.

Auerbach, S. (2006). Smart Game, Smart Toy. New York: St. Martin's Griffin.

Bates, B., (2003). Game Developer's Market Guide. Recuperado de http://www.batovi.com

Beck, K., et al. (2004). Extreme Programming Explained: Embrace Change (2nd Edition). Addison-Wesley Professional.

Björk, S. y Holopainen, J. (2005). Patterns in Game Design. Hingham: Charles River Media.

Castañeda, A. (2011). El Aprendiz. Barcelona: Endira.

Centro de Estudios Económicos Regionales [CEER] del Banco de la República. (2013). "Bilingüismo en Colombia". En: Documentos de trabajo sobre Economía Regional, n191, Colombia, capítulos 1-4.

Centro Virtual Cervantes. (s. f.). Respuesta Física Total. Recuperado de http://cvc.cervantes.es/ensenanza/biblioteca ele/diccio ele/diccionario/respue stafisicatotal.htm

Centro Virtual Cervantes. (s. f.). Enfoque Comunicativo. Recuperado de http://cvc.cervantes.es/ensenanza/biblioteca ele/diccio ele/diccionario/enfoqu ecomunicativo.htm

Consejo de Europa (2001). Marco Común Europeo de Referencia para las Lenguas: Aprendizaje, Enseñanza y Evaluación. Estrasburgo: Departamento de Política, Cultura y Deporte.

Crystal, D. (2003). English as a global language (4a Ed). Cambridge: Cambridge University Press.

Crytek. (2008). Transition to scrum midway through a development cycle: Lessons learned. In Game Developer Conference. 
Demachy T. (2008). Extreme game development. Recuperado de http://www.gamasutra.com/resource guide/20030714/demachy 01.shtml

Education First. (2014). The world's largest ranking of English skills. Recuperado de http://www.ef.edu/epi/

Egenfeldt-Nielsen, S. (2005). Beyond Edutainment: Exploring the Educational Potential of Games. Copenhagen: University of Copenhagen Press.

Fernández, J. (2013). Introducción a las metodologías ágiles. España.

Frasca, G. (2001). Videogames of the Oppressed: Videogames as a Means for Critical Thinking and Debate. Georgia: Institute of Technology.

Gamasutra. (2008). Interview: Nokia's scott foe - a member of the reset generation. Recuperado de http://www.gamasutra.com/php$\underline{\text { bin/news index.php?story }=19210}$

Gee, J. P. (2003). What Games Have to Teach Us about Learning. Oxford: Palgrave MacMillan.

Gonzalez, J. A. (s. f.). El lenguaje de programación C\#. Recuperado De http://dis.um.es/ bmoros/privado/bibliografia/LibroCsharp.pdf

Huizinga, J. (2000) Homo Ludens. Madrid: Alianza Editorial.

Juul, J. (2005). Half-Real: Video Games between Real Rules and Fictional Worlds. Cambridge: MIT Press.

Keith, C. (2007). Scrum rising. Game Developer Magazine.

Kniberg, H. (2007). Scrum Y Xp Desde Las Trinchera. Estados Unidos de América: C4Media

McFarlane, A. (2003). Report on the Educational Use of Games. Cambridge: Cambridge University Press.

Microsoft, (2003). Descripción de marcos por segundo (FPS). Recuperado de https://support.microsoft.com/en-us/kb/269068/es

Microsoft, (2012). Información del Registro de Windows para usuarios avanzados. Recuperado de https://support.microsoft.com/en-us/kb/256986/es 
Nutt, C. (2007). Living on the edge: Dice's owen o'brien speaks. Recuperado de http://www.gamasutra.com/view/feature/3684/living

Palacio, J. (2006). El modelo Scrum. Recuperado de http://www.navegapolis.net/files/s/NST-010 01.pdf

Ravaja, N.; Saari, T.; Turpeinen, M.; Laarni, J.; Salminen, M. y Kivikangas, M. (2005). "Spatial Presence and Emotions during Video Game Playing: Does it Matter with Whom You Play?” En: Teleoperators and Virtual Environments, vol 15, n4, USA, 381-392.

Rouse, R. (2001). Game Design: Theory \& Practice. Texas: Plano.

CEER. (2013). Banco de la República. Retrieved from http://www.banrep.gov.co/sites/default/files/publicaciones/archivos/dtser 191. pdf

Crystal, S. (2003). English as a global language. Cambridge University Press. 